\title{
Survival of ART restorations assessed using selected FDI and modified ART restoration criteria
}

\author{
Abeer Farag • Wil J. M. van der Sanden • \\ Hisran Abdelwahab • Jo E. Frencken
}

Received: 15 October 2009 /Accepted: 10 March 2010/Published online: 7 April 2010

(C) The Author(s) 2010. This article is published with open access at Springerlink.com

\begin{abstract}
A new set of criteria for assessing the quality of restorations using modern restorative materials, named FDI criteria, was recently introduced. This study tested the null hypothesis that there is no significant difference in survival estimate percentages of ART restorations assessed using selected FDI and modified ART criteria after 1 and 5 years. One operator placed a total of 60 class I and 30 Class II high-viscosity glass-ionomer ART restorations in ninety 14- to15-year-olds. Two calibrated and independent evaluators using both criteria evaluated restorations on diestone replicas at baseline and after 1 and 5 years. Statistical analyses were done using the Kaplan-Meier method and log-rank test. The survival results of ART restorations assessed using both sets of criteria after 1 and 5 years $(p=0.27)$ did not differ significantly. Three ART restorations were assessed as failures according to the ART criteria, while they were assessed as survived
\end{abstract}

\footnotetext{
A. Farag

Department of Conservative Dentistry, Faculty of Dentistry,

Minia University,

Minia, Egypt

W. J. M. van der Sanden

Department of Preventive and Restorative Dentistry,

Radboud University Nijmegen Medical Centre,

College of Dental Sciences,

Nijmegen, The Netherlands

H. Abdelwahab

Faculty of Dentistry, Ain Shams University,

Cairo, Egypt

J. E. Frencken $(\square)$

Department of Global Oral Health,

Radboud University Nijmegen Medical Centre,

College of Dental Sciences,

P. O. Box 9101, 6500 HB Nijmegen, The Netherlands

e-mail: j.frencken@dent.umcn.nl
}

using the FDI criteria. We conclude that the modified ART criteria enable reliable assessment of ART restorations in permanent teeth from diestone replicas and that there was no significant difference in survival estimates of ART restorations assessed using both sets of criteria. The null hypothesis was accepted.

Keywords Atraumatic restorative treatment . FDI evaluation criteria $\cdot$ Restoration assessment criteria . Restoration survival · ART criteria · Glass ionomer

\section{Introduction}

Criteria for assessing the clinical performance of restorations need to be reliable, pragmatic and easy to use. During the last three decades, most researchers have used the Ryge criteria for evaluating different restoration materials. However, researchers have over time adapted these criteria, in an effort to make them more discriminating in relation to modern restorative materials. Consequently, the alterations resulted in the need for names identifying particular sets of criteria; such as modified Ryge criteria or modified United States Public Health Service (USPHS) criteria [1].

As part of the development of the ART approach, more than two decades ago, restoration and sealant assessment criteria were created for use with ART [2]. In that developmental stage, special attention was given to the expected weakness of the restorative material used then for ART (medium-viscosity glass ionomer). Also regarded as extremely important was the need for reliable application of the criteria by researchers throughout the world, to facilitate reliable restoration survival comparison of this new caries management approach. 
The original set of ART restoration criteria has, meanwhile, been modified, as the first survival studies did not reveal the anticipated substantial level of restoration surface wear [3, 4]. Most studies investigating ART restoration survival have used the original and the modified ART restoration assessment criteria [5]. A few studies have used both the USPHS and the ART criteria to assess restoration quality. One study found that both criteria were comparable [6], while Lo et al. [7] concluded that the ART criteria were more stringent than those of the USPHS. The suggestion by Lo et al. [7] supports the conclusion of investigators who claim that the Ryge/USPHS criteria have limited sensitivity for detecting the improved clinical performance of the restorative materials currently in use [1]. In many cases, the insensitivity of the Ryge criteria is misinterpreted as good clinical performance [1].

Therefore, in order to detect early deterioration and differences between restorations using modern restorative materials, a new discriminative set of criteria, named FDI criteria, was developed [1]. It focuses on the clinical performance of the restorative materials, including aesthetic, functional and biological properties, and would allow for a more detailed analysis of failures.

As the FDI evaluation criteria have recently been introduced, they need to be compared to other existing restorative assessment criteria. The current study tests the null hypothesis that there is no significant difference in survival estimate percentages of ART restorations when assessed using selective FDI and the modified ART criteria after 1 and 5 years.

\section{Materials and methods}

The clinical study was carried out among 90 secondary students with a mean age of 14.6 years in Minia City, Egypt. Students were examined clinically for dental caries status at the school compound by the first author (AF), using the criteria developed by WHO. The ethics committee, comprising representatives of Minia University, Minia Local Government, Ministry of Education, Government Health Insurance and students' parents from Minia City, approved the study.

Implementation ART treatment was performed by the first author (AF) in the well-equipped clinic of the Dental School of Minia University, from November 2001 to March 2002. The operator did not have any prior experience in placing ART restorations and had acquired its principles and directions from reading the literature. In order to simulate a field setting, only the dental chair, spittoon and operating light were used. No chairside assistant was available. A junior staff member assisted in the administration of the study and, before the treatment started, individually instructed all students on how best to clean their teeth.

Treatment procedure The main study was intended to determine whether the use of chlorhexidine solution prior to restoration of the cleaned cavity would result in higher restoration survival rates. Therefore, half of the cavities were first disinfected for $1 \mathrm{~min}$ with a $2 \%$ chlorhexidine solution, Consepsis (Ultradent, South Jordan, USA), before being conditioned with Cavity Conditioner (GC, Leuven, Belgium). The treatment procedures used are described in detail in a previous publication [8].The size of cavity opening after completion of cavity cleaning was measured with a graded periodontal probe and rated as being smaller or larger than half the width of the occlusal tooth surface in both mesial-distal and buccal-lingual/palatal directions.

\section{Evaluation}

The restorations were evaluated at baseline, after 1 and 5 years. Photographs were taken immediately after the restoration was completed and subsequently after 1 year, using a colour film camera with a macro lens (Zenit12 KMZ, Moscow, Russia) and after 5 years, using a digital intraoral camera (Sopro 575, Acteon Group, La Ciotat, France). Impressions were taken of all restored teeth at all three evaluation periods, using a vinyl polysiloxane impression material, Affinis ${ }^{\mathrm{TM}}$ putty and light body (Coltène/Whaledent, Altstätten, Switzerland) in sectional impression trays, according to the manufacturer's instructions. Impressions were cast in high-strength, low-expansion dental stone, Type IV, Glastone (Dentsply, York, USA), capable of reproducing fine details up to $50 \mu \mathrm{m}$ [9].

Table 1 ART evaluation criteria used to assess ART restorations

\begin{tabular}{ll}
\hline Code & Criteria \\
\hline 0 & Present, satisfactory \\
1 & $\begin{array}{c}\text { Present, slight deficiency at cavity margin of less than } \\
0.5 \mathrm{~mm}^{\mathrm{a}}\end{array}$ \\
2 & $\begin{array}{l}\text { Present, deficiency at cavity margin of } 0.5 \mathrm{~mm} \text { or more } \\
\end{array}$ \\
3 & Present, fracture in restoration \\
4 & Present, fracture in tooth \\
5 & Present, overextension of approximal margin of $0.5 \mathrm{~mm}$ \\
6 & or more \\
7 & Not present, most or all of restoration missing \\
8 & Not present, other restorative treatment performed \\
9 & Unable to diagnose \\
$\mathrm{C}$ & Dentine carious lesion present
\end{tabular}

${ }^{\mathrm{a}}$ As assessed using the $0.5-\mathrm{mm}$ ball end of a metal CPI probe 


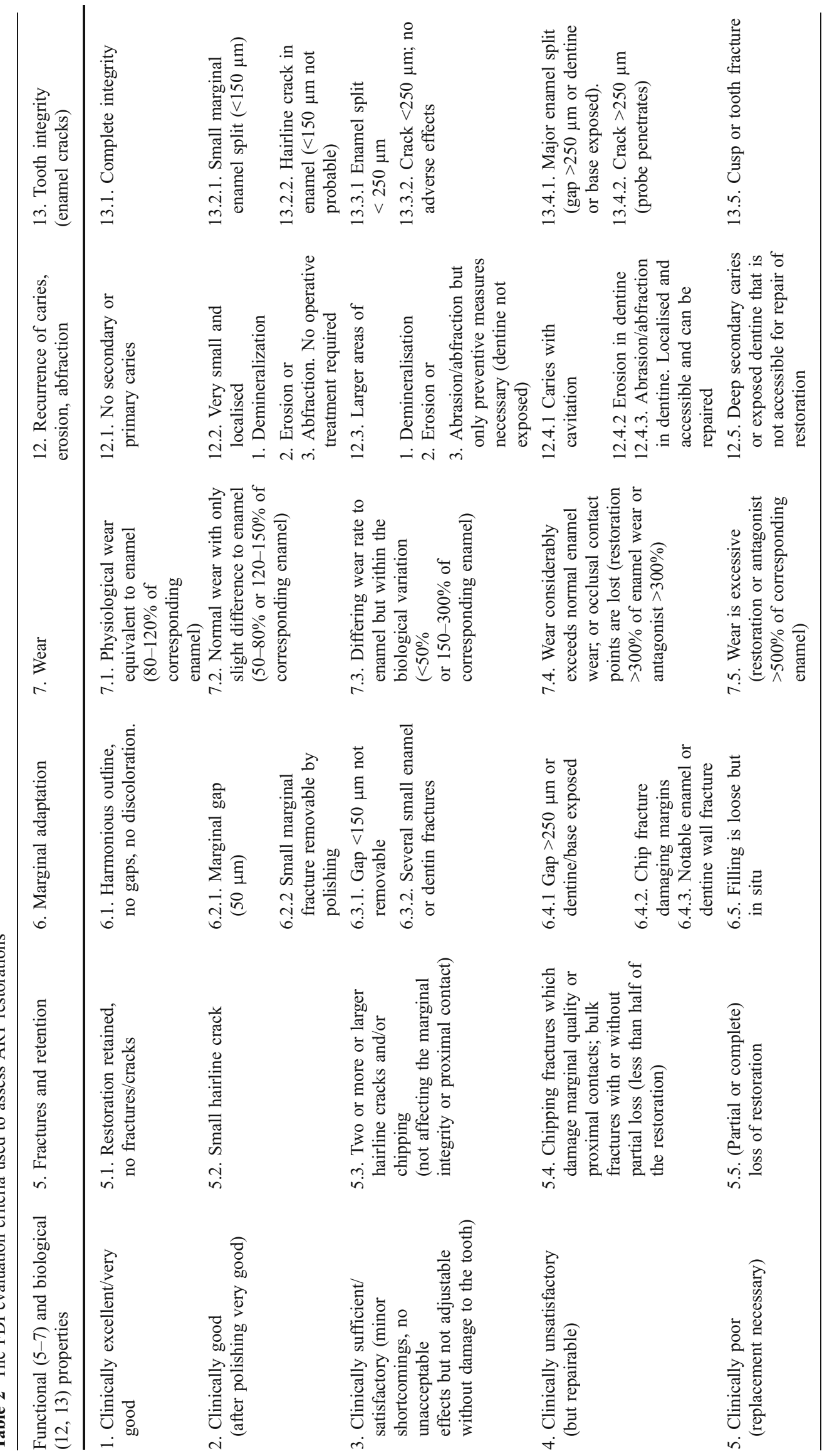


The restorations were evaluated using the replicas according to the ART criteria (Table 1) and the selected restoration characteristics from the newly developed FDI criteria (Table 2). If doubt arose, photographs were used to assist in making a judgment. In applying the ART criteria, deficiencies at the restoration margin were measured, using the $0.5-\mathrm{mm}$ ball-ended CPI probe (Ash/Dentsply, Addlestone, UK). A dentine carious lesion was scored 'present' if the CPI probe could penetrate the lesion during the clinical evaluation. Restorations with codes 0 and 1 were considered as having survived, while codes $2-7$ and ' $C$ ' were considered failures according to the ART criteria. As the assessment was performed extra-orally, not all features of the FDI criteria could be scored. Two specially designed probes with diameters of 150 and $250 \mu \mathrm{m}$ (Deppeler, Rolle, Switzerland) were used to measure deficiencies at the restoration margin, using the FDI criteria. The ICDAS criteria were used in the restored teeth for diagnosing the presence of carious lesions [10]. A cavitated dentine carious lesion was scored if a CPI probe could enter the cavity, during the clinical evaluation. According to the FDI criteria, restorations with codes 1,2 and 3 are considered as having survived, whereas codes 4 and 5 indicate a failure.

Two experienced and calibrated evaluators, one from the Netherlands (WS) and one from Egypt (HA), conducted the evaluation. They were involved in neither the planning of the study nor its execution. The inter-evaluator consistency tests for assessing restoration survival and diagnosing dentine carious lesions, expressed as kappa coefficient and percentage-observed $\left(P_{\text {obs }}\right)$ agreement value, were carried out using all replicas at evaluation years 1 and 5. The Landis and Koch criteria [11] were used to quantify the kappa test outcomes. Differences in assessments between evaluators were discussed and consensus was reached on all initial differences.
Statistical analysis Data were analysed, using SAS version 9.1. The independent variables were gender, mean DMFT score at baseline, restoration size (small/large), cavity type (single/multiple surface) and disinfected cavity (yes/no). The dependent variable was restoration survival. The Kaplan-Meier method was used in estimating the survival percentages. The log-rank test was applied in testing the null hypothesis and the effect of the independent variables on the restoration survival percentages. The level of significance was set at $\alpha=0.05$.

\section{Results}

Quality of the data The results of the inter-examiner consistency tests using ART and selected FDI criteria are presented in Table 3 . As no $2 \times 2$ contingency table could be constructed for the survival of ART restorations at evaluation year 1, restoration survival scores of evaluation years 1 and 5 were presented as combined. The reliability of assessing the FDI criteria properties fractures and retention, marginal adaptation and tooth integrity was found to be substantial. It was found fair for the property wear while moderate for diagnosing dentine carious lesions. The percentage of observed agreement was very high for all the properties of both assessment criteria, ranging from 0.92 to 1.00 .

Before reaching consensus, the evaluators differed in 51 of 242 of the judgements (21\%) using the ART criteria over the three evaluation periods. The differences predominantly concerned codes 0 and 1 . In only five judgements (three cases) did the difference have an effect on the survival outcome: four times the consensus score resulted in 'failure of the restoration' and once in 'success'. Four of the five judgements concerned the diagnosis of dentine carious lesions, which resulted in three failures.

Table 3 Inter-evaluator consistency assessments using kappa coefficient, its standard error (SE) and percentage correct observed scores $\left(P_{\text {obs }}\right)$ for assessing restoration failure (yes/no) and diagnosing dentine carious lesions by evaluation year using the selected FDI and modified ART criteria

\begin{tabular}{|c|c|c|c|c|c|c|c|c|c|c|c|c|}
\hline \multirow[t]{3}{*}{ FDI criteria } & \multicolumn{12}{|c|}{ Evaluation year } \\
\hline & \multicolumn{4}{|l|}{1} & \multicolumn{4}{|l|}{5} & \multicolumn{4}{|c|}{1 and 5 combined } \\
\hline & $N$ & Kappa & SE & $P_{\text {obs }}$ & $N$ & Kappa & SE & $P_{\mathrm{obs}}$ & $N$ & Kappa & SE & $P_{\mathrm{obs}}$ \\
\hline Fracture & 86 & 1.0 & 0.0 & 1.0 & 62 & 0.82 & 0.13 & 0.97 & 148 & 0.83 & 0.12 & 0.99 \\
\hline Marginal adaptation & 86 & 1.0 & 0.0 & 1.0 & 62 & 0.73 & 0.18 & 0.97 & 148 & 0.74 & 0.17 & 0.99 \\
\hline Wear & 86 & 1.0 & 0.0 & 1.0 & 62 & 0.38 & 0.28 & 0.95 & 148 & 0.39 & 0.28 & 0.98 \\
\hline Integrity & 85 & 1.0 & 0.0 & 1.0 & 62 & 0.79 & 0.20 & 0.98 & 147 & 0.80 & 0.20 & 0.99 \\
\hline $\begin{array}{l}\text { Restoration survival } \\
\text { ART criteria }\end{array}$ & 85 & 1.0 & 0.0 & 1.0 & 62 & 0.84 & 0.11 & 0.98 & 147 & 0.85 & 0.10 & 0.99 \\
\hline $\begin{array}{l}\text { Restoration survival } \\
\text { ART/FDI }\end{array}$ & 87 & N/A & & & 62 & 0.80 & 0.11 & 0.95 & 149 & 0.68 & 0.12 & 0.96 \\
\hline Dentine carious lesion & 85 & 1.0 & 0.0 & 1.0 & 61 & 0.50 & 0.19 & 0.92 & 146 & 0.53 & 0.18 & 0.97 \\
\hline
\end{tabular}


Using the FDI criteria, the evaluators differed in six of 242 judgements for fractures and retention, in 27 of 242 judgements for marginal adaptation, in 13 of 242 judgements for wear and in five of 242 judgements for tooth integrity, before reaching consensus. In only six judgements (four cases) differences had an effect on the survival outcome; four times the consensus score resulted in success and twice (two cases) in failure of the restoration. These latter two cases, each for fractures/retention and wear, had no failure scores for the other three restoration properties.

Disposition of restorations A total of 90 ART restorations were placed in 90 students: 57 males and 33 females, having a mean age of 14.6 years. There were 60 class I and 30 class II cavities restored in molar (93\%) and premolar (7\%) teeth. Maxillary teeth were restored in 22 of 90 and mandibular teeth in 68 of 90 of the subjects. The mean DMFT score was $6.7 \pm 2.3$ (standard deviation).

Restoration survival The restorations evaluated at 1 and 5 years by gender, cavity type, restoration size and treatment procedure, by the ART and selected FDI criteria, are presented in Table 4. Three restorations were excluded from analysis, as no replicas at evaluation year 1 were available.

The cumulative survival percentages and standard errors for all ART restorations assessed using the ART criteria were $97 \%(\mathrm{SE}=2.0 \%)$ at evaluation year 1 and $82 \%(\mathrm{SE}=4.7 \%)$ at evaluation year 5 (Table 4$)$. The corresponding figures for ART restorations assessed using the selected FDI criteria were $99 \%(\mathrm{SE}=1.1 \%)$ at evaluation year 1 and $86 \%(\mathrm{SE}=4.4 \%)$ at evaluation year 5 . Only one significant effect was observed between the dependent and the independent variables in data assessed by ART and selected FDI criteria at 5 years. This concerned a gender effect $(p=0.03)$ for ART restorations assessed according to the ART criteria.

A total of 12 restorations failed: three at evaluation year 1 and nine at evaluation year 5, according to the ART criteria. According to the FDI criteria, nine restorations failed: one at evaluation year 1 and eight at evaluation year 5 (Table 5). All the three ART restorations that failed using the ART criteria, and those that survived using the FDI criteria scored code 2 in relation to the ART criteria.
Table 4 Cumulative survival $(\%)$ and standard error (SE) of ART restorations assessed using the modified ART and selected FDI criteria after 1 and 5 years by gender, cavity type, restoration size and procedure

\footnotetext{
$P$ value is per independent variable

$N$ number of restorations placed, cl 1 black class 1 restoration, $\mathrm{cl}$ 2 black class 2 restoration

${ }^{\text {a }}$ Statistically significant
}

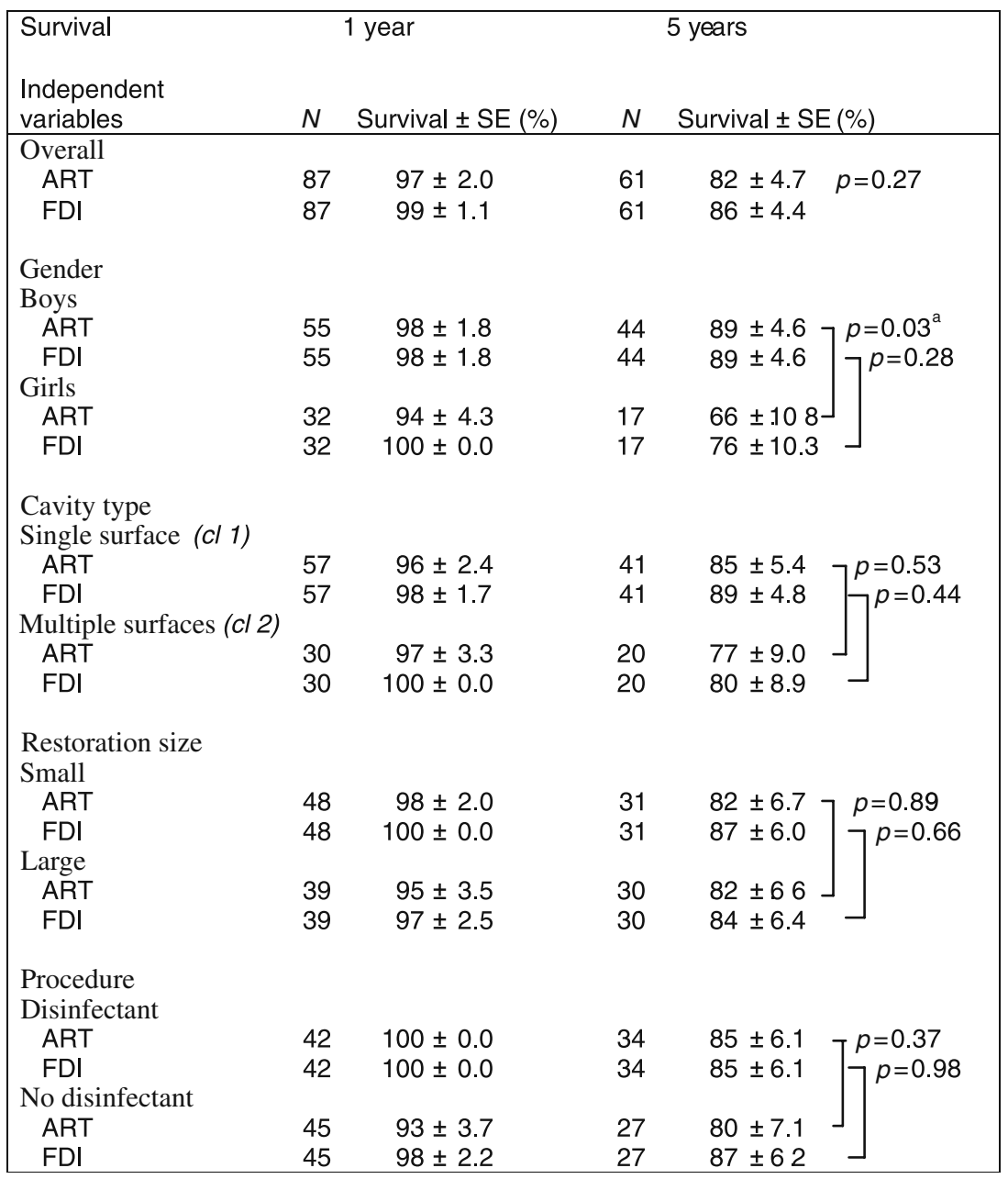


Table 5 Failed ART restorations according to the ART and FDI criteria at 1 and 5 years' evaluation

\begin{tabular}{|c|c|c|c|c|c|c|}
\hline \multirow{2}{*}{$\begin{array}{l}\text { Tooth } \\
\text { number }\end{array}$} & \multirow{2}{*}{$\begin{array}{l}\text { ART } \\
\text { criteria }\end{array}$} & \multicolumn{5}{|c|}{ FDI criteria } \\
\hline & & Fracture & Adaptation & Wear & Caries & $\begin{array}{l}\text { Tooth } \\
\text { integrity }\end{array}$ \\
\hline
\end{tabular}

\begin{tabular}{|c|c|c|c|c|c|c|}
\hline \multicolumn{7}{|c|}{1 year } \\
\hline 37 & 6 & $\mathrm{~F}$ & $\mathrm{~s}$ & $\mathrm{~s}$ & $\mathrm{~s}$ & $\mathrm{~s}$ \\
\hline 16 & 2 & $s$ & $s$ & $s$ & $s$ & $s$ \\
\hline 37 & 2 & $s$ & $s$ & $s$ & $s$ & $s$ \\
\hline \multicolumn{7}{|c|}{5 years } \\
\hline 36 & 4 & $\mathrm{~F}$ & $\mathrm{~F}$ & $\mathrm{~F}$ & $\mathrm{~F}$ & $\mathrm{~F}$ \\
\hline 36 & 3 & $\mathrm{~s}$ & $\mathrm{~s}$ & $\mathrm{~F}$ & $\mathrm{~s}$ & $\mathrm{~s}$ \\
\hline 36 & $\mathrm{C}$ & $\mathrm{s}$ & s & $\mathrm{s}$ & $\mathrm{F}$ & $\mathrm{s}$ \\
\hline 26 & 2 & $\mathrm{~F}$ & $\mathrm{~s}$ & $\mathrm{~s}$ & $\mathrm{~s}$ & $\mathrm{~s}$ \\
\hline 47 & $\mathrm{C}$ & $\mathrm{s}$ & $\mathrm{s}$ & $\mathrm{s}$ & $\mathrm{F}$ & $\mathrm{s}$ \\
\hline 36 & 2 & $s$ & $s$ & $s$ & $s$ & $s$ \\
\hline 16 & 3 & $\mathrm{~F}$ & $\mathrm{~F}$ & $\mathrm{~s}$ & $\mathrm{~F}$ & $\mathrm{~s}$ \\
\hline 37 & $\mathrm{C}$ & $\mathrm{s}$ & $\mathrm{s}$ & $\mathrm{s}$ & $\mathrm{F}$ & $\mathrm{s}$ \\
\hline 16 & 3 & $\mathrm{~F}$ & $\mathrm{~F}$ & $\mathrm{~s}$ & $\mathrm{~F}$ & $\mathrm{~s}$ \\
\hline
\end{tabular}

Rows in italics refer to restorations that were assessed a failure using the ART criteria and that survived using the FDI criteria

$s$ successful, $F$ failure, $C$ dentine carious lesion

\section{Discussion}

The percentage of correctly observed judgements $\left(P_{\text {obs }}\right)$ was used to complement the Kappa coefficient reliability measurement. This was necessary as the kappa statistic is unreliable in low prevalence populations such as the present one. As all $P_{\text {obs }}$ readings and most of the kappa coefficients were high, it was concluded that the quality of the data obtained was high.

In the process of using the ART approach to restore an occlusal cavity, both the cavity and the pits and fissures are filled. An ART restoration is, therefore, a sealant restoration [2]. This implies that restorative material, usually a highviscosity glass ionomer, is placed not only in the cavity but also very frequently in contiguous pits and fissures of occlusal surfaces, adjacent to the cavity. In this aspect, ART restorations differ from resin composite or amalgam restorations, which aim to finish the restoration at the cavity margin, leaving pits and fissures unfilled.

It is known that glass ionomer, like other directly placed restorative materials, deteriorates over time. Deterioration is particularly noticeable in glass-ionomer sealants [12]. In a number of cases in the present study, the sealant part of the glass-ionomer ART restoration had clinically disappeared, leaving an inverse step visible towards the restoration part of the ART restoration. This condition, considered by the evaluators to be either a marginal gap (failure) or not (success), was the main reason influencing their disagree- ment about the survival outcome. However, an inverse step at the cavity margin may also be mistaken as a true marginal gap, exposing enamel and/or dentine. Such difficulties in using the ART criteria have not been reported before and were most probably due to sufficient time available for assessing the ART restorations from replicas and discussing different outcomes between the two evaluators. In future glass-ionomer ART studies, evaluators should pay attention to the phenomenon of the inverted step when assessing ART restorations in occlusal surfaces. The presence of an inverse step is not considered a failure.

The survival results of ART restorations assessed in accordance with both assessment criteria after 1 and 5 years did not differ statistically significantly. A difference was apparent in three ART restorations that were assessed as 'failure' according to the ART criteria and as sound according to the selected FDI criteria. All three ART restorations had a score of 2 , which means that more than $0.5 \mathrm{~mm}$ of enamel was visible clinically at any part of the cavity wall. The FDI criteria for marginal adaptation accept the total length of visible enamel as sound and only considers a marginal gap of $>0.25 \mathrm{~mm}$ or exposed dentine/base as a condition for restoration failure [1]. Using the earlier Ryge criteria, only exposed dentine/base would be a reason for failing a restoration in the category 'marginal integrity' [13]. The ART criteria for marginal integrity (codes 1 and 2) consider a marginal deficiency of $\geq 0.5 \mathrm{~mm}$ to denote a failed restoration. As code 2 of the ART criteria assesses not only marginal gap formation but also surface wear, it is difficult to compare the outcomes of marginal adaptation using both sets of criteria strictly. Therefore, the outcome of the present study shows that the ART criteria are comparable to the selected FDI criteria in assessing ART restoration survival in permanent teeth.

Another difference between the two sets of criteria used in the present study is the absence of extracted restored and re-restored teeth in the database of the survival analyses using the FDI criteria. The same is also applicable when the ART criteria are compared to the Ryge criteria. As the survival of ART restorations in the present study was based on the restoration assessment outcomes from replicas only, the absence of re-restored and of extracted restored teeth had no influence on the final outcome. However, including or excluding extracted restored teeth and re-restored teeth does make a difference in survival percentages of restorations. It is, therefore, safe to say that if the ART restorations in the published literature had been assessed using the Ryge criteria, the reported survival of ART restorations would have been higher.

The present study has shown that the modified ART criteria are a reliable measurement instrument for assessing ART restoration survival. The criteria are far less detailed 
and include far fewer clinical restoration characteristics than the FDI criteria but are easier and faster to use. It is therefore recommended that the ART criteria be used for restoration and tooth survival assessment in clinical oral health services studies and that the FDI criteria be used for studying the quality of restorative materials only [1].

In conclusion, the ART criteria gave reliable assessment of ART restorations in permanent teeth from replicas, and there was no significant difference in survival estimates of ART restorations assessed using both sets of criteria. The null hypothesis was accepted.

Acknowledgements The first author is very grateful to Dr. Fayez Mohammed Hassan, Dr. Elham Moustafa Fawzy and Dr. Mushira Dahaba for the assistance received during the implementation of the study. We thank Mr. J. Mulder for analysing the data.

Conflict of interest The authors declare that they have no conflict of interest.

Open Access This article is distributed under the terms of the Creative Commons Attribution Noncommercial License which permits any noncommercial use, distribution, and reproduction in any medium, provided the original author(s) and source are credited.

\section{References}

1. Hickel R, Roulet JF, Bayne S, Heintze SD, Mjör IA, Peters M, Rousson V, Randall R, Schmalz G, Tyas M, Vanherle G (2007)
Recommendations for conducting controlled clinical studies of dental restorative materials. Clin Oral Invest 11:5-33, Erratum published in Vol 12, p97

2. Frencken JE, Pilot T, Songpaisan Y, Phantumvanit P (1996) Atraumatic Restorative Treatment (ART): rationale, technique and development. J Public Health Dent 56:135-140

3. Frencken JE, Makoni F, Sithole WD (1998) ART restorations and glass ionomer sealants in Zimbabwe: survival after 3 years. Community Dent Oral Epidemiol 26:372-381

4. Smales RJ, Gao W, Ho FT (1997) In vitro evaluation of sealing pits and fissures with newer glass ionomer cements developed for the ART technique. J Clin Pediatr Dent 21:321-323

5. Hof MA Van 't, Frencken JE, van Palenstein Helderman WH, Holmgren CJ (2006) The ART approach for managing dental caries: a meta-analysis. Int Dent J 56:345-351

6. Holmgren CJ, Lo ECM, Hu DY, Wan HC (2000) ART restorations and sealants placed in Chinese school children - result after three years. Community Dent Oral Epidemiol 28:314-320

7. Lo ECM, Luo Y, Fan MW, Wei SHY (2001) Clinical Investigation of two glass ionomer restoratives used with atraumatic restorative treatment approach in china: two years results. Caries Res 35:458-463

8. Farag A, van der Sanden WJM, Abdelwahab H, Mulder J, Frencken JE (2009) 5-Year survival of ART restorations with and without cavity disinfection. J Dent 37:468-474

9. Graig RG, Powers JM (2002) Restorative dental materials, 11th edn. Mosby, St. Louis, p 397

10. Ismail AI, Sohn W, Tellez M, Amaya A, Sen A, Hasson H, Pitts NB (2007) The international caries detection and assessment system (ICDAS): an integrated system for measuring dental caries. Community Dent Oral Epidemiol 35:170-178

11. Landis JR, Koch GG (1977) The measurement of observer agreement for categorical data. Biometrics 33:159-174

12. Frencken JE, Wolke J (2010) Clinical and SEM assessment of ART high-viscosity glass-ionomer sealants after $8-13$ years in 4 teeth. J Dent 38:59-64

13. Ryge G (1980) Clinical criteria. Int Dent J 30:347-358 